\title{
Fatores associados à prática insuficiente de atividade física em professores escolares brasileiros: um estudo de revisão integrativa
}

\section{Factors associated with insufficient physical activity practice in Brazilian school teachers: an integrative review study}

\author{
Factores asociados con la actividad física \\ insuficiente entre los docentes escolares brasileños:
}

un estudio de revisión integradora

Gildiney Penaves de Alencar ${ }^{1}$

Fabiana Maluf Rabacow ${ }^{2}$

Alexandra Maria Almeida Carvalho ${ }^{3}$

${ }^{1}$ Mestre em Saúde e Desenvolvimento na Região Centro-Oeste pela Universidade Federal de Mato Grosso do Sul (UFMS). Graduado em Educação Física pela Universidade Católica Dom Bosco (UCDB). Membro do Centro de Estudos e Pesquisas em Atividade Física e Saúde (CEPAFS/UFMS). Professor da Rede Municipal de Ensino de Campo Grande (REME) e docente do curso de Educação Física do Centro Universitário da Grande Dourados (UNIGRAN/EAD). E-mail: gildiney.gpa@gmail.com, ORCID: https://orcid.org/0000-0002-5177-495X

${ }^{2}$ Doutora em Medicina Preventiva pela Universidade de São Paulo (USP). Mestre em Educação Física pela Universidade Federal de Santa Catarina (UFSC). Graduada em Educação Física pela Universidade Federal de Mato Grosso do Sul (UFMS).

Coordenadora e docente do curso de Educação Física da Universidade Católica Dom Bosco (UCDB). E-mail: rf3227@ucdb.br, ORCID: https://orcid.org/0000-0001-5315-4650

${ }^{3}$ Doutora em Biosystems Eng. and Environmental Science pela University of Tennessee

(UT). Mestre em Engenharia Elétrica pela Universidade Federal de Uberlândia (UFU). Graduada em Engenharia Civil pela Universidade Estadual Paulista Júlio de

Mesquita Filho (UNES). Professora assistente da Universidade Federal de Mato Grosso do Sul (UFMS) e coordenadora do Programa de Pós-Graduação em Saúde e Desenvolvimento na Região Centro-Oeste. E-mail: profalexandraufms@gmail.com,

ORCID: https://orcid.org/0000-0002-7868-3214 
Resumo: A prática de atividade física é importante na prevenção de doenças crônicas, e sua investigação é válida para estimular hábitos saudáveis entre os professores. O objetivo deste estudo foi identificar os fatores associados à prática insuficiente de atividade física em professores escolares brasileiros, por meio de uma revisão integrativa de artigos publicados de 2009 a 2019 nas bases de dados Lilacs, SciELO, DOAJ, PubMed, Dialnet e Web of Science, através das combinações dos descritores exatos e sinônimos em português e em inglês, pelos termos "Professores Escolares", "Atividade Física" e "Brasil". A maioria dos professores são insuficientemente ativos e, entre os fatores associados a esta condição, estão o excesso de peso, massa corporal elevada, maior percentual de gordura, dor crônica, problemas relacionados à voz, tabagismo e fatores ocupacionais, como o excesso de trabalho, contrato temporário, falta de autonomia e o tempo em pé.

Palavras-chave: atividade motora; professores escolares; Brasil.

\begin{abstract}
The practice of physical activity is important in the prevention of chronic diseases, and its investigation is valid to encourage healthy habits among teachers. This study aimed to identify factors associated with insufficient physical activity in Brazilian school teachers through an integrative review of articles published from 2009 to 2019 in the Lilacs, SciELO, DOAJ, PubMed, Dialnet, and Web of Science databases, through combinations of the exact descriptors and synonyms in English and Portuguese, using the terms "School Teachers", "Physical Activity", and "Brazil". Most teachers are insufficiently active and, among the factors associated with this condition, are overweight, high body mass, a higher percentage of fat, chronic pain, problems related to voice, smoking, and occupational factors, such as overwork, temporary contract, lack of autonomy, and standing time.
\end{abstract}

Keywords: motor activity; school teachers; Brazil.

Resumen: La práctica de la actividad física es importante en la prevención de enfermedades crónicas y su investigación es válida para fomentar hábitos saludables entre los docentes. El objetivo de este estudio fue identificar los factores asociados con la actividad física insuficiente en los docentes de escuela brasileños a través de una revisión integradora de artículos publicados de 2009 a 2019 en las bases de datos Lilacs, SciELO, DOAJ, PubMed, Dialnet y Web of Science, a través de combinaciones de los descriptores exactos y sinónimos en portugués e inglés, con los términos "Maestros", "Actividad Física" y "Brasil". La mayoría de los maestros son insuficientemente activos $y$, entre los factores asociados con esta afección, se encuentran el sobrepeso, la alta masa corporal, el mayor porcentaje de grasa, el dolor crónico, los problemas relacionados con la voz, el tabaquismo y los factores laborales, como el exceso de trabajo, el contrato temporal, falta de autonomía y tiempo de espera.

Palabras clave: actividad motora; docentes; Brasil. 
Fatores associados à prática insuficiente de atividade física em professores escolares brasileiros: um estudo de revisão integrativa

\section{INTRODUÇÃO}

A docência é tida como uma ocupação que tem sua atuação nas mais diversas etapas de ensino, seja na educação básica, seja na educação superior ou em programas de pós-graduação. Tratando-se especificamente de professores que atuam em escolas, de acordo com dados mais atuais disponíveis, cerca de 2.212.018 docentes trabalhavam na educação básica brasileira, em 2019.

As características do trabalho docente e as funções exercidas exigem um alto comprometimento profissional e, na maior parte dos casos, resulta numa demanda exacerbada de atividades executadas em sala de aula quanto fora dela, com execução de planejamentos, reuniões, orientações que consomem muito tempo cotidianamente, o que gera reflexos negativos na saúde física e mental e leva o professor a adotar um estilo de vida irregular, além de contribuir para uma qualidade de vida ruim.

A falta da atividade física é apontada como um dos principais fatores de risco de morte no mundo, e sua prática regular é considerada uma ferramenta importante na prevenção de doenças crônicas. Investigar dados sobre hábitos de atividade física em professores escolares é uma estratégia válida para averiguar a situação atual e incentivar a realização de programas que estimulem a introduzir esta prática no cotidiano, já que, além de melhorar as condições de saúde, é possível aumentar também a satisfação, diminuir o estresse e reduzir o absenteísmo.

Porém o que se vê em nível mundial é que os professores relatam práticas insuficientes de atividade física, e esta atitude pode levar ao aparecimento de diversos problemas, como os sintomas osteomusculares, por exemplo. A realidade brasileira não se difere desta perspectiva, já que muitos profissionais inseridos na educação, especialmente os professores, não praticam atividade física e apresentam níveis baixos ou insuficientes, contribuindo para aumentar os riscos de desenvolver alguma doença crônica e até mesmo condições específicas, afastando-os do trabalho e das salas de aula.

Neste sentido, este estudo foi realizado com o objetivo de identificar quais são os fatores associados à prática insuficiente de atividade física em professores escolares brasileiros por meio de um estudo de revisão integrativa. 


\section{MATERIAIS E MÉTODOS}

A revisão integrativa é uma forma de revisão que se utiliza de estudos já publicados no meio científico e os resume, na intenção de possibilitar um maior e melhor entendimento acerca de determinado assunto. Neste estudo, utilizaram-se as recomendações propostas por Whittemore e Knafl (2005), quando se trata das cinco etapas da revisão integrativa (Figura 1).

Figura 1 - Etapas da revisão integrativa de acordo com Whittemore e Knafl (2005)

Etapa 1:

Identificação

do problema
Etapa 2:

Busca na

literatura
Etapa 3:

Avaliação dos dados
Etapa 4:

Análise dos dados
Etapa 5:

Apresentação dos resultados

Fonte: Elaboração dos autores.

Etapa 1 - Identificação do problema

O problema foi identificado por variáveis de interesse da questão norteadora "Quais são os fatores associados à prática insuficiente de atividade física em professores escolares brasileiros?".

Etapa 2 - Busca na literatura

A busca na literatura foi realizada por meio de descritores exatos do Medical Subjects Headings (MeSH), no inglês, sendo utilizados "School Teachers", "Brazil" e o sinônimo "Physical Activity"; e, da mesma forma, no português, os descritores exatos em Ciências da Saúde (DeCS) "Professores Escolares", "Brasil" e o sinônimo "Atividade Física". As buscas dos estudos 
Fatores associados à prática insuficiente de atividade física em professores escolares brasileiros:

deram-se por meio das combinações entre os descritores utilizando os Operadores Booleanos AND e OR.

As bases de dados utilizadas foram: Literatura Latino-Americana e do Caribe em Ciências da Saúde (Lilacs), Eletronic Library Online (SciELO), Directory of Open Access Journals (DOAJ), PubMed, Dialnet e Web of Science. As buscas nessas bases se fizeram por meio das combinações dos descritores exatos e sinônimos pelos seguintes termos: "School Teachers" AND "Physical Activity" AND "Brazil".

Foram incluídos artigos com texto completo disponível em meio on-line nos idiomas português, inglês e espanhol, de 2009 a 2019, que respondessem à questão norteadora deste estudo, e tiveram como público-alvo professores escolares brasileiros. Foram excluídos os estudos que não tinham o texto completo disponível em meio on-line, os escritos em outras línguas que não o inglês, português e espanhol, bem como os estudos que não responderam à questão norteadora e não tiveram como público-alvo professores escolares brasileiros.

Dos artigos encontrados, foi realizada a leitura do título, resumo e palavras-chave para a pré-seleção dos estudos. Os pré-selecionados foram lidos na íntegra, destes, os que respondiam à questão norteadora compuseram a amostra final deste estudo.

Etapa 3 - Avaliação dos dados

Para esta etapa, utilizou-se um instrumento de coleta de dados contendo as seguintes variáveis: base de dados, autor(es) e título do artigo, periódico e ano de publicação, tipo de estudo e natureza da instituição de ensino, objetivo e principais resultados.

Etapa 4 - Análise dos dados

Esta etapa resultou nas seguintes categorizações: caracterização da amostra; avaliação dos artigos; fatores relacionados à composição corporal; dores crônicas, problemas relacionados à voz e tabagismo; fatores ocupacionais. 
Etapa 5 - Apresentação dos resultados

Resultou na elaboração deste artigo, que apresenta de forma clara e concisa a revisão integrativa, colocando todas as etapas elaboradas, resultados segundo as variáveis (autor, ano; tipo de estudo, população, local, período de coleta, instrumento de medida de atividade física utilizado; objetivo, principais resultados), discussão e considerações finais.

\section{RESULTADOS}

\subsection{Caracterização da amostra}

As buscas nas bases de dados resultaram em 31 artigos, sendo: Lilacs (7 artigos), SciELO (10 artigos), DOAJ (2 artigos), PubMed (8 artigos), Dialnet (não obteve resultado) e Web of Science (4 artigos). Após aplicação dos filtros, pré-seleção com a leitura dos títulos, resumos, palavras-chave, critérios de inclusão e exclusão, foram selecionados 22 artigos que foram lidos na íntegra, sendo excluídos 14 deles, resultando em uma amostra final de 8 estudos (figura 1 ).

A base de dados SciELO, assim como a DOAJ e Web of Science, obtiveram os artigos repetidos em $100 \%$ da amostra, sendo excluídas, o que ocorreu com a base Dialnet, por não ter logrado resultados nas buscas realizadas. As bases Lilacs e PubMed foram as únicas com trabalhos na amostra final, com 5 e 3 artigos, respectivamente.

Figura 2 - Fluxograma da etapa de seleção dos artigos

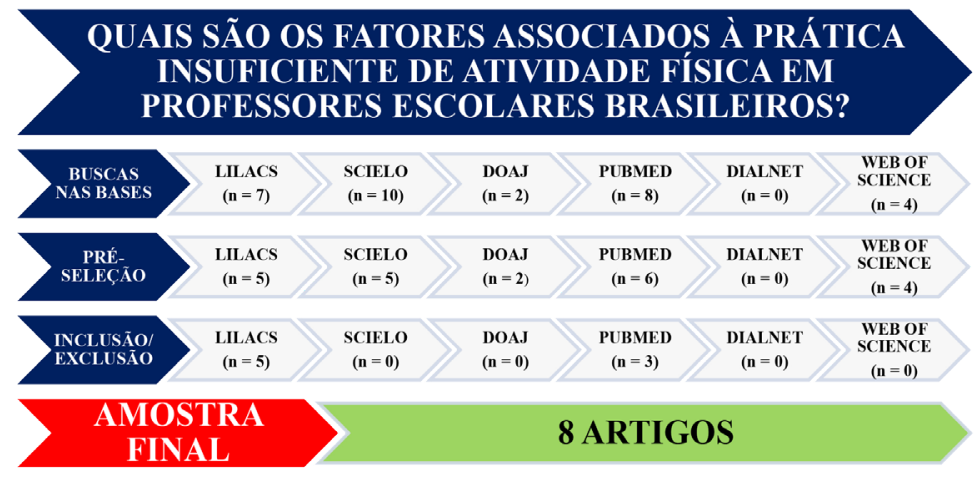

Fonte: Elaboração dos autores. 


\subsection{Avaliação dos artigos}

Aproximadamente $90 \%$ (7 artigos) dos estudos analisaram professores de escolas públicas (municipal e estadual) e quase $40 \%$ ( 3 estudos) de escolas particulares, sendo que dois estudos tiveram amostras de professores tanto de escolas públicas quanto de escolas privadas. No que se refere à região do Brasil onde os estudos foram produzidos, $75 \%$ foram conduzidos na região Sudeste e cerca de $40 \%$ na região Sul e Nordeste, mas vale o destaque em relação aos estudos de Barbosa e Fonseca (2019) e Santos et al. (2019), os quais realizaram suas pesquisas numa amostra representativa nacional e retrataram as cinco regiões geográficas brasileiras.

Os oito artigos que fizeram parte desta revisão integrativa foram avaliados de acordo com as seguintes variáveis: base de dados; autor(es) e título do artigo; periódico e ano de publicação; tipo de estudo e natureza da instituição de ensino; cidade e/ou região do Brasil onde o estudo foi realizado; objetivo; e principais resultados (Quadro 1). 


\begin{tabular}{|c|c|c|c|}
\hline 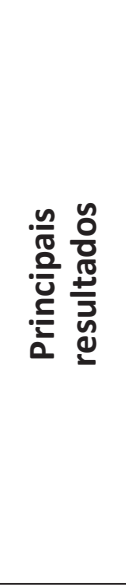 & 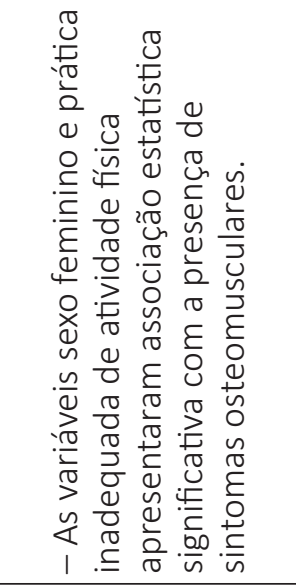 & 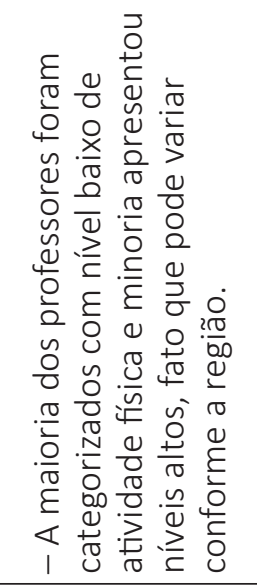 & 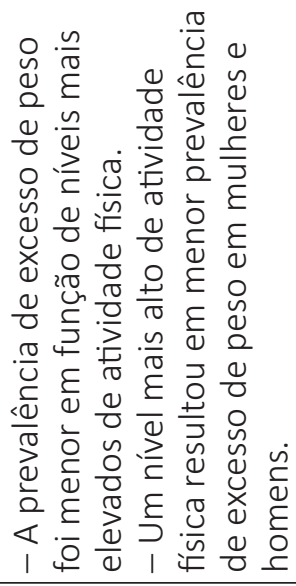 \\
\hline $\begin{array}{l}\stackrel{0}{\circ} \\
\frac{1}{0} \\
\frac{0}{0}\end{array}$ & 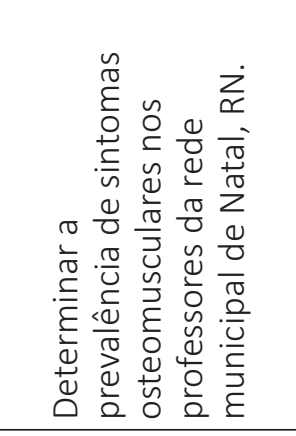 & 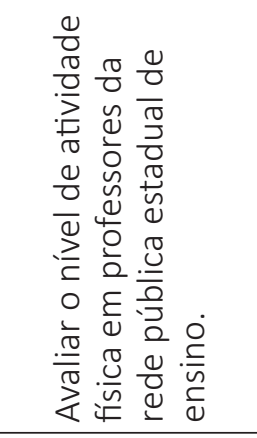 & 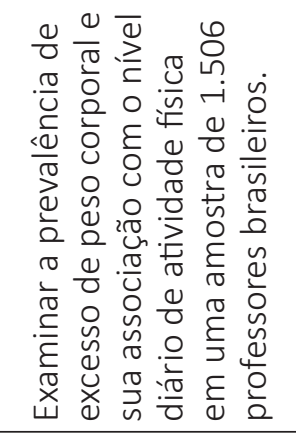 \\
\hline 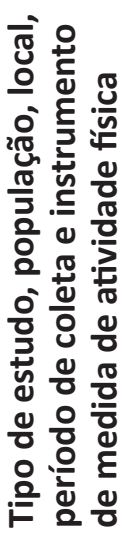 & 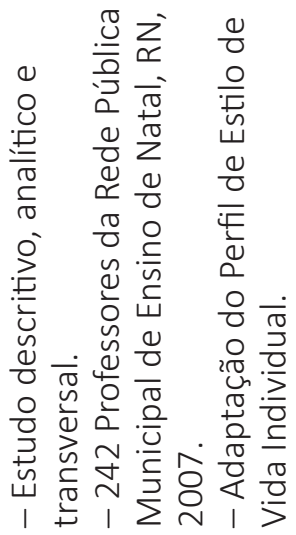 & 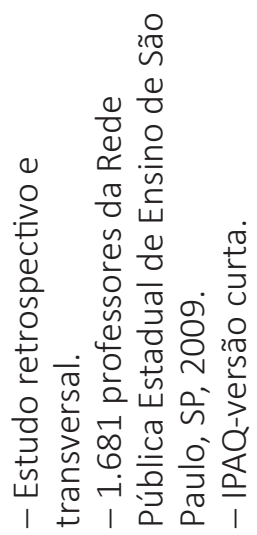 & 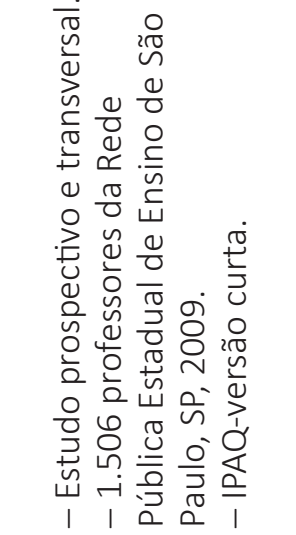 \\
\hline 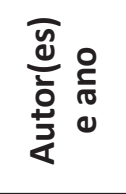 & 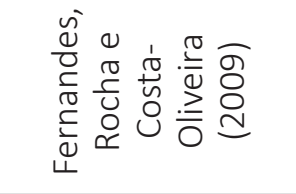 & 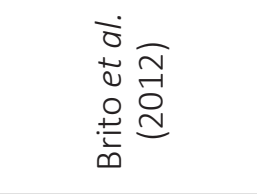 & 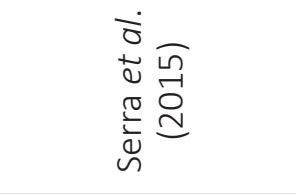 \\
\hline$\exists S \forall g$ & Sכㄱרור & SЈ $\forall 717$ & ałwgnd \\
\hline
\end{tabular}




\begin{tabular}{|c|c|c|}
\hline 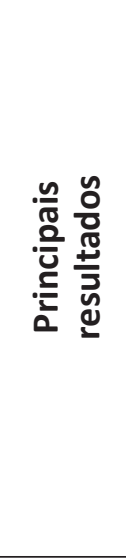 & 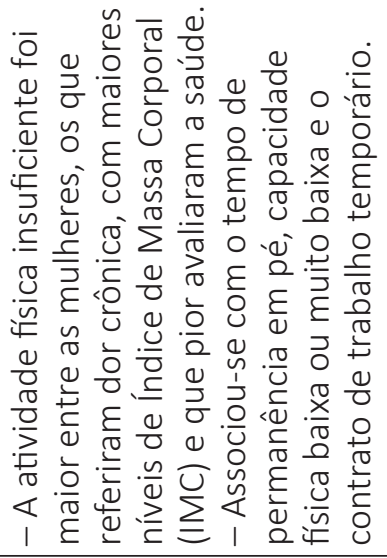 & 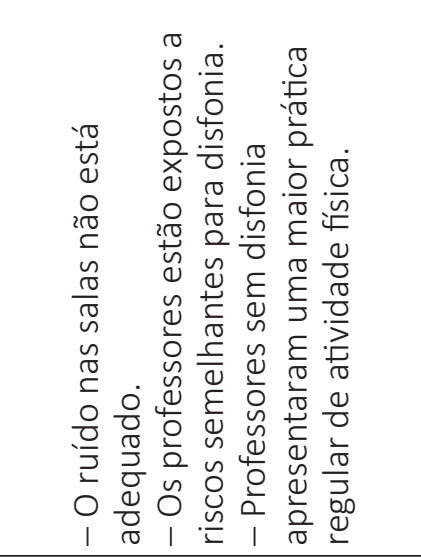 \\
\hline $\begin{array}{l}\frac{0}{7} \\
\frac{0}{0} \\
\frac{0}{0}\end{array}$ & 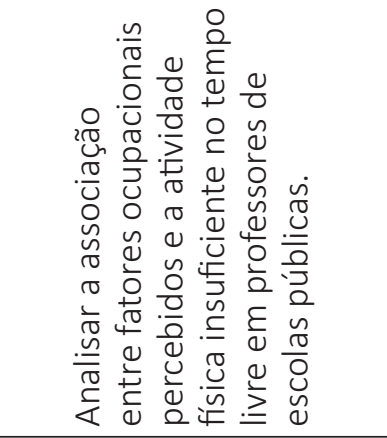 & 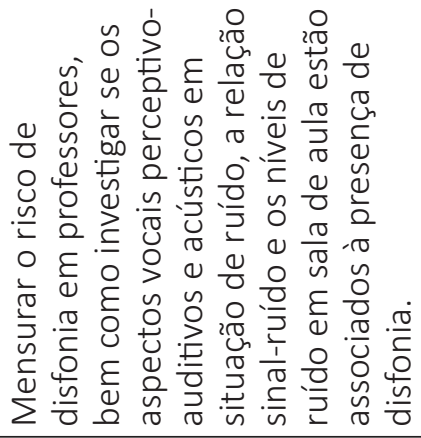 \\
\hline 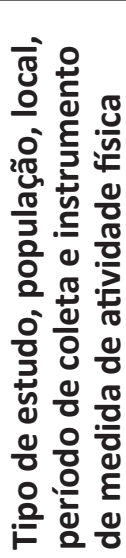 & 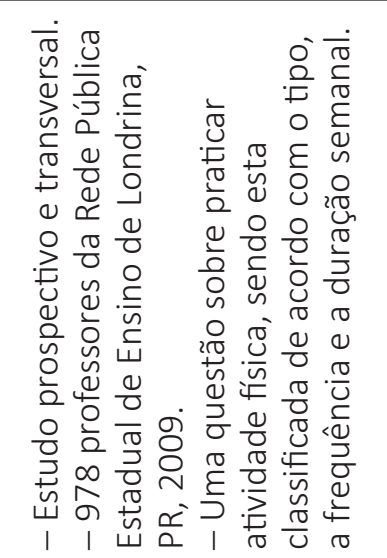 & 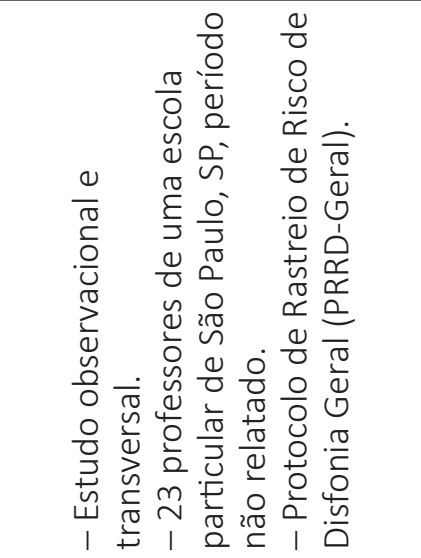 \\
\hline 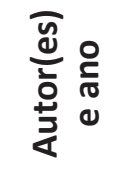 & 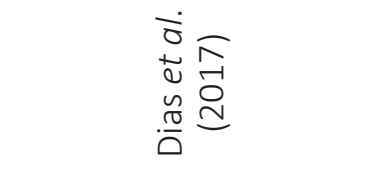 & 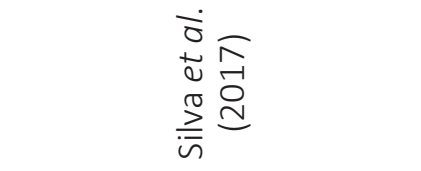 \\
\hline$\exists S \forall g$ & ałwgnd & Sכ $\forall ר ו 7$ \\
\hline
\end{tabular}




\begin{tabular}{|c|c|c|}
\hline 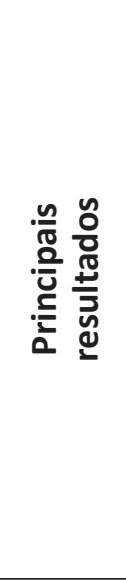 & 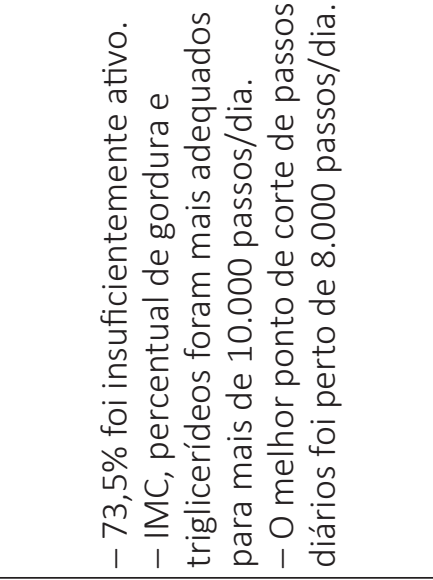 & 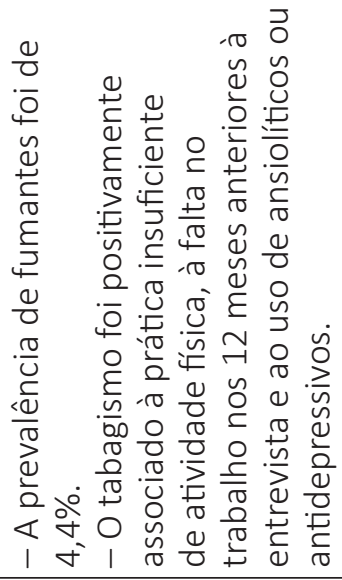 \\
\hline $\begin{array}{l}\frac{0}{2} \\
\frac{0}{0} \\
\frac{0}{0}\end{array}$ & 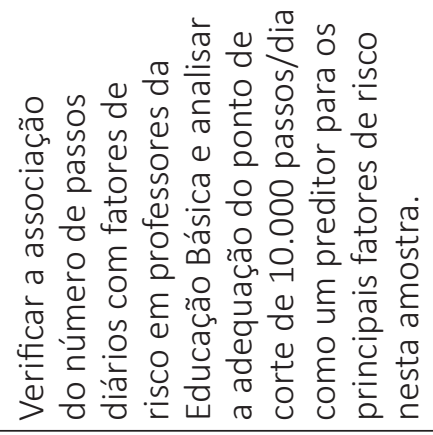 & 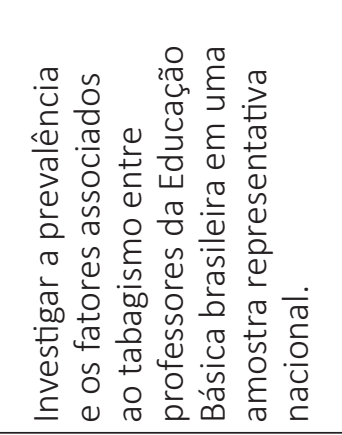 \\
\hline 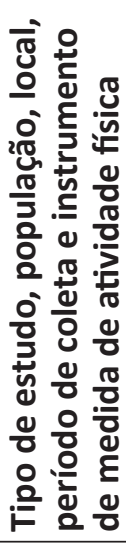 & 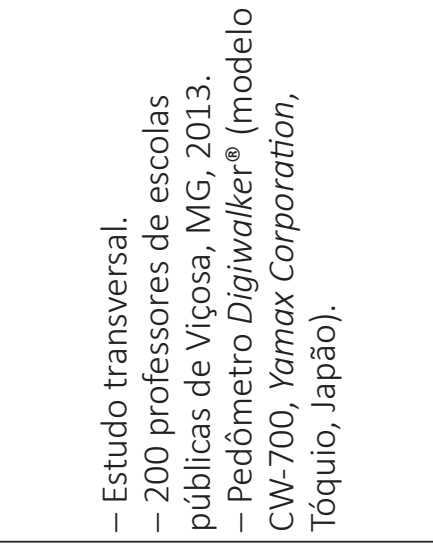 & 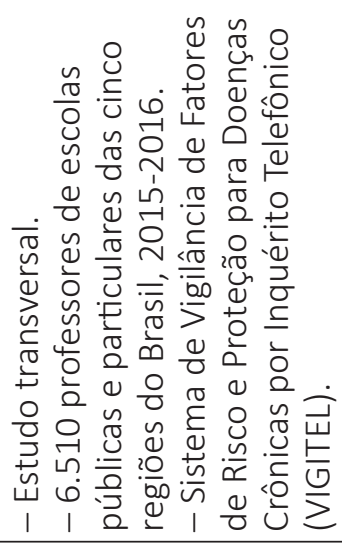 \\
\hline 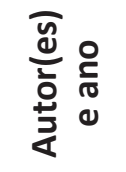 & 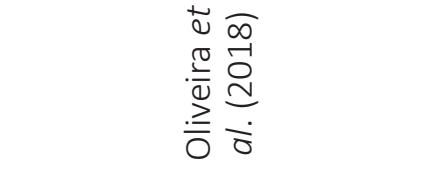 & 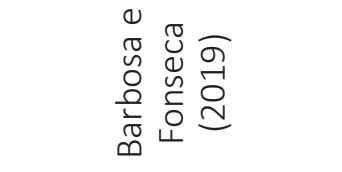 \\
\hline$\exists S \forall g$ & ałWgnd & Sכ $\forall 7 ו 7$ \\
\hline
\end{tabular}


Fatores associados à prática insuficiente de atividade física em professores escolares brasileiros: um estudo de revisão integrativa

\begin{tabular}{|c|c|}
\hline 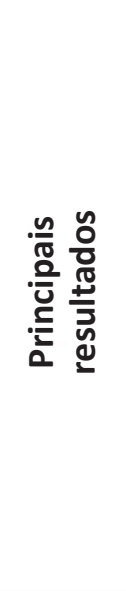 & 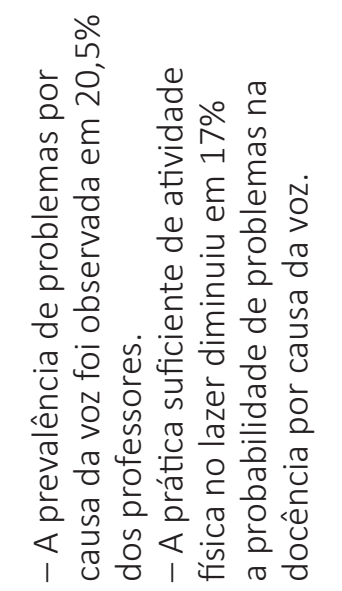 \\
\hline $\begin{array}{l}\frac{0}{2} \\
\frac{0}{0} \\
\frac{0}{0}\end{array}$ & 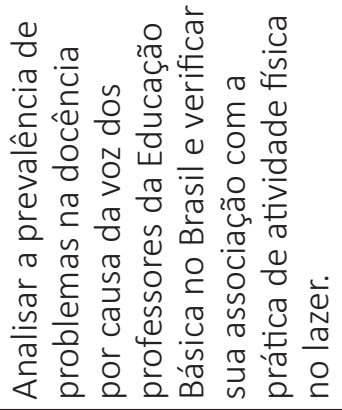 \\
\hline 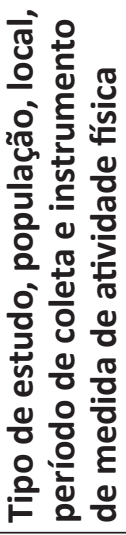 & 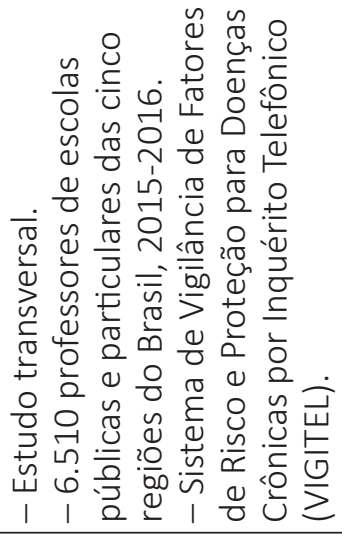 \\
\hline 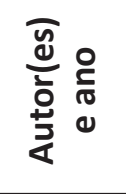 & 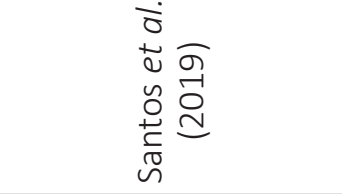 \\
\hline$\exists \mathbf{S} \forall \mathbf{g}$ & 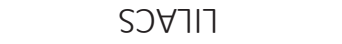 \\
\hline
\end{tabular}


Há uma predominância de mulheres no trabalho docente $(66,0 \%)$, com idade média de 40,9 anos, nos oito artigos que compuseram este estudo. Todos os artigos selecionados associaram a prática de atividade física com algum fator que impacta na saúde dos professores, mesmo que este objetivo tenha sido abordado em segundo plano. Entre estes fatores, a baixa adesão à atividade física esteve ligada a excesso de peso, uma elevada massa corporal e um maior percentual de gordura, presentes em $25 \%$ dos estudos ( $n=2)$, assim como $25 \%(n=2)$ dos artigos observaram relação entre a prática insuficiente de atividade física e a presença de dor crônica ou sintomas osteomusculares; $25 \%(n=2)$, problemas relacionados à voz; $12,5 \%$ $(n=1)$, tabagismo; e $12,5 \%(n=1)$, fatores ocupacionais, como o contrato de trabalho temporário.

Para mensurar a prática de atividade física dos professores, 62,5\% $(n=5)$ dos estudos utilizaram questionários como instrumentos; $25 \%(n=2)$, entrevista telefônica; e apenas um estudo (12,5\%) fez uso de pedômetro, estabelecendo 10.000 passos diários como o ponto de corte para que sujeito fosse considerado ativo (OLIVEIRA et al., 2018).

Dos cinco estudos que aplicaram questionários, 40\% ( $n=2)$ usaram a versão curta do International Physical Activity Questionnaire (IPAQ), sendo classificados quanto ao nível de atividade física (baixo, moderado e alto), e os estudos que utilizaram a entrevista telefônica fizeram uso do questionário do Sistema de Vigilância de Fatores de Risco e Proteção para Doenças Crônicas por Inquérito Telefônico (VIGITEL), sobre a prática de atividade física suficiente no lazer, considerando a modalidade para determinar a intensidade, duração e frequência, sendo que os professores que praticam atividade física por 150 minutos ou mais, semanalmente, foram considerados ativos, da mesma forma como foi visto nos outros estudos.

\section{DISCUSSÃO}

De forma geral, nota-se que os professores escolares brasileiros têm uma baixa adesão à prática de atividade física regular, com destaque para os resultados de Fernandes, Rocha e Costa-Oliveira (2009) e de Oliveira et al. (2018), em que obtiveram $80,7 \%$ e $73,5 \%$ de professores com práticas 
Fatores associados à prática insuficiente de atividade física em professores escolares brasileiros:

insuficientes de atividade física, respectivamente. Vale ressaltar que o primeiro estudo utilizou um questionário validado para a população brasileira, e o segundo, um pedômetro para estimar a quantidade de atividade física praticada diariamente.

De acordo com a Organização Mundial da Saúde, 150 minutos de atividade física moderada semanalmente é o recomendado para adultos com a intenção de aproveitar seus benefícios para a saúde e reduzir os riscos de doenças crônicas (WORLD HEALTH ORGANIZATION, 2020), e grande parte da população docente não consegue atingir esses níveis.

Diversos fatores estão associados à baixa adesão à atividade física regular, tais como fatores relacionados à composição corporal (excesso de peso corporal, massa corporal elevada, maior percentual de gordura), dor crônica, problemas relacionados à voz, tabagismo e fatores ocupacionais, como a alta carga horária em sala de aula, o tipo de contrato de trabalho e o tempo de permanência em pé.

\subsection{Fatores relacionados à composição corporal}

Conforme Du et al. (2013) e Machado et al. (2014) mencionam, o baixo nível de atividade física está ligado a um excesso de peso, de massa corporal e de percentual de gordura, tanto na população mundial quanto na população brasileira, e nos artigos encontrados com o público docente não foi diferente. No estudo de Oliveira et al. (2018), os autores tiveram o objetivo de verificar a associação do número de passos diários com fatores de risco e mostraram que níveis adequados de Índice de Massa Corporal (IMC), percentual de gordura e triglicerídeos foram encontrados no grupo que realizou pelo menos 10.000 passos diários, ponto de corte considerado adequado. Já o excesso de peso e dislipidemia estiveram associados à redução do número de passos.

Dias et al. (2017) detectaram também que mais da metade dos professores com maiores níveis de IMC eram considerados inativos ou insuficientemente ativos. Serra et al. (2015) encontraram resultados parecidos que sustentam tal fato. Os autores descobriram que, entre os 1.506 professores avaliados, $25,3 \%(n=382)$ apresentaram excesso de peso e 
um nível de atividade física considerado baixo. Além disso, foi identificado que a prevalência de pessoas com excesso de peso foi menor em função de maiores níveis de atividade física, e maiores níveis de atividade física resultaram em menor prevalência de sobrepeso para homens e mulheres, respectivamente (SERRA et al., 2015).

Levando em consideração o sexo, um nível mais alto de atividade física resultou em menor prevalência de excesso de peso tanto para homens como para mulheres ( $p=0,005)$ (OLIVEIRA et al., 2018). Serra et al. (2015) relataram que os valores de IMC adequados foram notados nos homens classificados com nível de atividade física moderado ( $O R=1,69 ; p=0,030)$ e alto $(O R=2,57 ; p=0,002)$, enquanto para as mulheres essa associação foi percebida naquelas que tiveram um nível moderado de atividade física $(\mathrm{OR}=1,43, p=0,004)$, ou seja, realizam pelo menos 3 dias ou mais de atividade física com intensidade vigorosa, por 20 minutos diários.

Com tais resultados, é possível notar que se torna importante a prática de atividade física regular, já que é considerado um tratamento não medicamentoso e uma ação preventiva eficaz, além de ser um mecanismo de controle de peso, regulação de gordura corporal e de prevenção de doenças cardiovasculares, itens importantes quando se trata da saúde dos professores (WORLD HEALTH ORGANIZATION, 2010; THE EUROPEAN SOCIETY OF CARDIOLOGY, 2012; GHORAYEB et al., 2013).

\subsection{Dores crônicas, problemas relacionados à voz e tabagismo}

O baixo nível de atividade física também esteve ligado ao aparecimento de dores crônicas ou sintomas osteomusculares, caracterizados como doenças em que há ocorrência de sintomas como dor, parestesias, fadiga, que acometem principalmente os membros superiores (SATO et al., 1993). Dias et al. (2017) avaliaram 978 professores de ensino fundamental e médio, dos quais $42 \%$ relataram dor crônica e, entre estes, $76,6 \%$ eram inativos ou insuficientemente ativos fisicamente.

Fernandes, Rocha e Costa-Oliveira (2009) constataram 93\% de prevalência de sintomatologia osteomuscular na rede municipal de ensino de Natal, RN, e 47,7\% tiveram de se afastar da atividade docente. Quando 
Fatores associados à prática insuficiente de atividade física em professores escolares brasileiros:

realizado o teste de associação, notaram que a prática inadequada de atividade física $(p=0,037)$ foi uma das variáveis que apresentaram associação estatística significativa com a presença de sintomas osteomusculares, ou seja, a falta de atividade física regular influencia no aparecimento de dores osteomusculares (FERNANDES; ROCHA; COSTA-OLIVEIRA, 2009).

De acordo com o Ministério da Saúde, professores e trabalhadores da educação estão entre as profissões que mais têm fatores de risco para desenvolver sintomas osteomusculares (BRASIL, 2001), o que impacta diretamente na qualidade de vida desses profissionais (KARAKAYA et al., 2015), acarretando o afastamento das salas de aula (FERNANDES; ROCHA; COSTA-OLIVEIRA, 2009).

A baixa adesão a programas de atividade física regular esteve também associada com problemas relacionados à voz em professores escolares, como descreve Silva et al. (2017). Neste estudo, os autores avaliaram 23 professoras de Educação Infantil e Ensino Fundamental, que foram divididas em dois grupos, sem disfonia e com disfonia; observou-se um maior risco de desenvolver problemas vocais no grupo com disfonia, que também teve uma menor aderência à prática de atividade física (SILVA et al., 2017).

Bassi et al. (2011) e Mello et al. (2013) relatam que a realização de atividade física regular promove menos estresse, tensão corporal e tensão vocal, isso pelo fato de que praticantes de atividade física tendem a apresentar uma melhor aptidão física e cardiorrespiratória (ARAÚJO; ARAÚJO, 2000), o que é benéfico na produção da voz.

Dados recentes apresentados por Santos et al. (2019, p. 5) mostram que a "prática suficiente de atividade física no lazer diminuiu em 19\% a probabilidade de problemas na docência por causa da voz entre os professores da Educação Básica do Brasil”, e a frequência semanal de 3 a 4 dias ou 5 dias ou mais "também apresentou associação inversa aos problemas de voz", o que evidencia a importância da atividade física no dia a dia dos docentes escolares.

Outro fator que esteve também presente nesta revisão integrativa foi o tabagismo, associado à prática insuficiente de atividade física no estudo Barbosa e Fonseca (2019), mesmo que a prevalência de fumantes tenha sido reduzida (4,4\%). Os autores investigaram 6.510 professores da Educação Básica 
brasileira e observaram uma menor prevalência de fumantes entre os professores que eram suficientemente ativos (3,4\%) (BARBOSA; FONSECA, 2019).

Silva et al. (2017, p. 7) ressaltam que os "hábitos de vida são estabelecidos pelo indivíduo", e o que foi visto na pesquisa de Barbosa e Fonseca (2019) pode vir ao encontro desta afirmação, já que a atividade física e o tabagismo são hábitos que o próprio indivíduo pode decidir. Além disso, para aqueles que já têm o hábito saudável e que conhecem os benefícios desse comportamento, a tendência é de que seja menor a predisposição de fazer uso do tabaco (CARDOSO et al., 2010).

\subsection{Fatores ocupacionais}

Fatores ocupacionais afetam também diretamente na prática de atividade física por professores, conforme foi percebido nos estudos selecionados. Fernandes, Rocha e Costa-Oliveira (2009) revelam que a alta carga horária em sala de aula, somada ao tempo despendido em atividades extraclasse, como atividades domésticas e correção de avaliações, por exemplo, explica o motivo da baixa participação em atividades físicas regulares, o que interfere também no seu estado de saúde e na produtividade (SANTOS et al., 2019).

O excesso de trabalho (SILVA et al., 2011; HUSE et al., 2020) e, consequentemente, o cansaço físico (VIEIRA; SILVA, 2019) representam uma importante barreira na realização de atividade física entre brasileiros. Corroborando estes dados, Dias et al. (2017) constataram que 20,9\% dos professores trabalham acima de 40 horas semanais, o que vem sendo apresentado também por Santos et al. (2019) e Barbosa e Fonseca (2019), em que detectaram que 56,2\% e 58,8\% dos professores brasileiros trabalham por 40 horas semanais ou mais, respectivamente. Estes achados evidenciam o fato de grande parte dos professores escolares estarem insuficientemente ativos.

Outro item destacado nos estudos foi o tipo de contrato de trabalho estabelecido nas relações de emprego entre os professores. Dias et al. (2017), ao entrevistarem 978 professores de Londrina, Paraná, identificaram que a atividade física insuficiente no tempo livre esteve associada ao tipo de 
Fatores associados à prática insuficiente de atividade física em professores escolares brasileiros:

contrato, sendo que a prevalência entre os temporários (76,5\%) foi maior que os estatutários (69,8\%); porém, apesar da diferença, uma alta prevalência foi também encontrada nos professores concursados.

O fato de os professores temporários serem considerados menos ativos em relação aos professores estatutários se deve aos locais de atuação destes docentes, já que, no começo do ano letivo, os professores estatutários escolhem em qual escola atuar e geralmente optam por lugares mais próximos geograficamente, enquanto os temporários ficam com as vagas remanescentes mais distantes (DIAS et al., 2017), o que também interfere nos hábitos de atividade física (BRITO et al., 2012). Além deste quesito, os temporários são lotados em locais com piores condições de trabalho, sendo expostos a fatores precários, como o ruído elevado, indisciplina em sala de aula e a falta de autonomia (BARBOSA; FONSECA, 2019; SANTOS et al., 2019), que podem ser prejudiciais para a saúde e a qualidade de vida desses docentes (MILANI; FIOD, 2008) - algo observado também em professores de ensino superior (BOSI, 2007).

O tempo de permanência em pé durante a atividade laboral foi ainda apontado como um motivo do afastamento às atividades físicas, visto o cansaço produzido pela posição ortostática e o fato de considerarem que já realizam um esforço físico, não sendo necessário efetuar mais esforço além deste (DIAS et al., 2017).

Quanto aos componentes curriculares ministrados nas escolas, descobrimos que o motivo de lecionar Educação Física e ter os conhecimentos mais específicos sobre as vantagens da atividade física sobre a saúde não é totalmente decisivo para este comportamento se tornar um hábito, mesmo que os professores de Educação Física tenham uma prevalência de atividade física insuficiente menor em comparação aos professores de outras disciplinas (DIAS et al., 2017).

Considerar os aspectos laborais para a realização de programas que incentivam a prática de atividades físicas voltadas aos professores é importante, mas é necessário também compreender que este é um comportamento influenciado por múltiplos fatores, conforme Bauman et al. (2012) salientam. Porém ações são fundamentais para diminuir os riscos aos quais estes profissionais são expostos, e considerar os fatores associados à baixa adesão 
de atividade física por professores escolares, aqui apresentados, pode ser uma estratégia eficaz de promover hábitos saudáveis entre esta população.

Assim, além das benfeitoras alcançadas pela prática regular de atividade física na saúde docente, tendo menos gastos com tratamentos e redução no número de afastamentos, é um investimento interessante não somente aos professores, mas à população discente e à administração em geral.

\section{CONSIDERAÇÕES FINAIS}

Dado o exposto neste estudo, percebe-se que a maioria dos professores escolares brasileiros são insuficientemente ativos fisicamente, e diversos são os fatores que estão associados a este comportamento, tais como o excesso de peso corporal, uma massa corporal elevada, maior percentual de gordura, dor crônica, problemas relacionados à voz, tabagismo e fatores ocupacionais.

Entre os fatores ocupacionais, os achados demonstram que o excesso de trabalho devido à alta carga horária em sala e em atividades extraclasse estão entre os motivos que mais afastam os professores de praticarem atividade física no momento de lazer. Além destes, o tipo de contrato temporário, ruído elevado, indisciplina, falta de autonomia e o tempo em pé parecem gerar um cansaço adicional que impedem os docentes de sentirem vontade em realizar alguma atividade física.

O fato de lecionar Educação Física não isenta da situação de ser considerado insuficientemente ativo, mesmo que a prevalência de atividade física insuficiente dos professores deste componente curricular tenha sido menor em relação aos professores de outras áreas.

Outros fatores ainda precisam ser identificados para que possam ser permanentemente acompanhados, a fim de fundamentar ações para reduzir os riscos aos quais os professores são expostos e que estimulem hábitos saudáveis no ambiente escolar, entre eles, a prática de atividade física regular.

\section{REFERÊNCIAS}

ARAÚJO, D. S. M. S.; ARAúJO, C. G. S. Aptidão física, saúde e qualidade de vida relacionada à saúde em adultos. Revista Brasileira de Medicina do Esporte, São Paulo, v. 6, n. 5, p. 194-203, 2000. doi: http://dx.doi.org/10.1590/ S1517-86922000000500005 
Fatores associados à prática insuficiente de atividade física em professores escolares brasileiros: um estudo de revisão integrativa

BARBOSA, R. E. C.; FONSECA, G. C. Prevalência de tabagismo entre professores da Educação Básica no Brasil, 2016. Cadernos de Saúde Pública, Rio de Janeiro, v. 35, supl. 1, p. 1-15, 2019. doi: https://doi.org/10.1590/0102-311X00180217

BASSI, I. B.; ASSUNÇÃO, A. A.; GAMA, A. C. C.; GONÇALVES, L. G. Características clínicas, sociodemográficas e ocupacionais de professoras com disfonia. Revista Distúrbios da Comunicação, São Paulo, v. 23, n. 2, p. 173-180, 2011.

BAUMAN, A. E.; REIS, R. S.; SALLIS, J. F.; WELLS, J. C.; LOOS, R. J.; MARTIN, B. W. Correlates of physical activity: why are some people physically active and others not? The Lancet, Londres, v. 380, n. 9838, p. 258-71, 2012. doi: https://doi. org/10.1016/S0140-6736(12)60735-1

BOSI, A. P. Precarização do trabalho docente nas instituições de ensino superior do Brasil nesses últimos 25 anos. Educação e Sociedade, Campinas, v. 28, n. 101, p. 1503-23, 2007.

BRASIL. Ministério da Saúde. Departamento de Ações Programáticas e Estratégicas. Área Técnica de Saúde do Trabalhador. Lesões por Esforços Repetitivos (LER) e Distúrbios Osteomusculares Relacionados ao Trabalho (DORT). Brasília-DF, 2001.

BRITO, W. F.; SANTOS, C. L.; MARCOLONGO, A. A.; CAMPOS, M. D.; CAMPOS, M. D.; BOCALINI, D. S.; ANTONIO, E. L.; SILVA JUNIOR, J. A.; TUCCI, P. J. F.; SERRA, A. J. Nível de atividade física em professores da rede estadual de ensino. Revista de Saúde Pública, São Paulo, v. 46, n. 1, p. 104-9, 2012. doi: https://doi.org/10.1590/ S0034-89102012000100013

CARdoso, D. B.; COElho, A. P.; ROdRigues, M.; PetrolanU, A. Fatores relacionados ao tabagismo e ao seu abandono. Revista de Medicina, São Paulo. v. 89, n. 2, p. 76-82, 2010.

DIAS, D. F.; LOCH, M. R.; GONZÁLEZ, A. D.; ANDRADE, S. M.; MESAS, A. E. Atividade física insuficiente no tempo livre e fatores ocupacionais em professores de escolas públicas. Revista de Saúde Pública, São Paulo, v. 51, n. 68, p. 1-10, 2017. doi: https:// doi.org/10.1590/S1518-8787.2017051006217

DU, H.; BENNETT, D.; LI, L.; WHITLOCK, G.; GUO, Y.; COLLINS, R.; CHEN, J.; BIAN, Z.; HONG, L. S.; FENG, S.; CHEN, X.; CHEN, L.; ZHOU, R.; MAO, E.; PETO, R.; CHEN, Z. Physical activity and sedentary leisure time and their associations with $\mathrm{BMI}$, waist circumference, and percentage body fat in 0.5 million adults: the China Kadoorie Biobank study. The American Journal of Clinical Nutrition, Rockville, v. 97, n. 3, p. 487-96, 2013. doi: https://doi.org/10.3945/ajcn.112.046854 
FERNANDES, M. H.; ROCHA, V. M. R.; COSTA-OLIVEIRA, A. G. R. Fatores associados à prevalência de sintomas osteomusculares em professores. Revista de Salud Pública, Bogotá, v. 11, n. 2, p. 256-67, 2009. doi: https://doi.org/10.1590/ S0124-00642009000200010

GHORAYEB, N.; COSTA, R. V. C.; CASTRO, I.; DAHER, D. J.; OLIVEIRA FILHO, J. A.; OLIVEIRA, M. A. B. et al. Diretriz em Cardiologia do Esporte e do Exercício da Sociedade Brasileira de Cardiologia e da Sociedade Brasileira de Medicina do Esporte. Arquivos Brasileiros de Cardiologia, Rio de Janeiro, v. 100, n. 1, supl. 2, p. 1-41, 2013. doi: https://doi.org/10.5935/abc.2013S002

HUSE, O.; PALERMO, C.; EVANS, M.; PEETERS, A. Factors influencing healthy eating and physical activity amongst school staff. Health Promotion International, Londres, v. 35, n. 1, p. 123-31, 2020. doi: https://doi.org/10.1093/heapro/day100

KARAKAYA, I. Ç.; KARAKAYA, M. G.; TUNÇ, E.; KIHTIR, M. Musculoskeletal problems and quality of life of elementar school teachers. International Journal of Occupational Safety and Ergonomics, Londres, v. 21, n. 3, p. 344-50, 2015. doi: https://doi.org/10.1080/10803548.2015.1035921

MACHADO, Í. E.; PEREIRA, S. C. L.; DIAS JÚNIOR, C. S.; ABREU, M. N. S.; BORGES, A. M.; FILGUEIRAS, J. H. Fatores associados ao excesso de peso em adultos usuários de restaurantes populares em Belo Horizonte, Brasil. Ciência e Saúde Coletiva, Rio de Janeiro, v. 19, n. 5, p. 1367-77, 2014. doi: https://doi. org/10.1590/1413-81232014195.21772013

MELLO, M. T.; LEMOS, V. A.; ANTUNES, H. K. M.; BITTENCOURT, L.; SANTOS-SILVA, R.; TUKIF, S. Relationship between physical activity and depression and anxiety symptoms: a population study. Journal of Affective Disorders, Amsterdam, v. 149, n. 1-3, p. 241-6, 2013. doi: https://doi.org/10.1016/j.jad.2013.01.035

MILANI, N. Z.; FIOD, E. G. M. Precarização do trabalho docente nas escolas públicas do Paraná (1990-2005). Roteiro, Joaçaba, v. 33, n. 1, p. 77-100, 2008.

OLIVEIRA, R. A. R.; AMORIM, P. R. S.; MOTA JÚNIOR, R. J.; TAVARES, D. D. F.; FARIA, F. R.; MOREIRA, O. C.; LIMA, L. M.; BRITTO, R. R.; MARINS, J. C. B. Association between the number of daily steps and the cardiovascular risk factors in basic education teachers. Journal of Sports Medicine and physical fitness, Torino, v. 58, n. 5, p. 71420, 2018. doi: https://doi.org/10.23736/S0022-4707.17.07330-3

SANTOS, S. M. M.; MAIA, E. G.; CLARO, R. M.; MEDEIROS, A. M. Limitação do uso da voz na docência e a prática de atividade física no lazer: Estudo Educatel, Brasil, 
Fatores associados à prática insuficiente de atividade física em professores escolares brasileiros: um estudo de revisão integrativa

2015/2016. Cadernos de Saúde Pública, Rio de Janeiro, v. 35, supl. 1, p. 1-12, 2019. doi: https://doi.org/10.1590/0102-311X00188317

SATO, L.; ARAÚJO, M. D.; UDIHARA, M. L.; FRANCO, M. A.; NICOTERA, F. N.; DALDON, M. T.; SETTIMI, M. M.; SILVESTRE, M. P. Atividades em grupo com portadores de L.E.R. e achados sobre a dimensão psicossocial. Revista Brasileira de Saúde Ocupacional, São Paulo, v. 21, n. 79, p. 49-62, 1993.

SERRA, A. J.; BRITO, W. F.; ANTONIO, E. L.; SANTANA, E. T.; BOCALINI, D. S.; SANTOS, L. F. N.; DEANA, A. M.; RAMBO, C. S. M.; CARVALHO, P. T. C.; SILVA JUNIOR, J. A.; TUCCI, P. J. F. To be or not to be physically active: Insights for a real chance to have an appropriate body mass in a sample of teachers. Work: A Journal of Prevention, Assessment \& Rehabilitation, Amsterdam, v. 52, n. 2, p. 441-6, 2015. doi: https:// doi.org/10.3233/WOR-152130

SILVA, B. G.; CHAMMAS, T. V.; ZENARI, M. S.; MOREIRA, R. R.; SAMELLI, A. G.; NEMR, $K$. Análise de possíveis fatores de interferência no uso da voz durante atividade docente. Revista de Saúde Pública, São Paulo, v. 51, n. 124, p. 1-10, 2017. doi: https://doi.org/10.11606/S1518-8787.2017051000092

SILVA, S. G.; SILVA, M. C.; NAHAS, M. C.; VIANA, S. L. Fatores associados à inatividade física no lazer e principais barreiras na percepção de trabalhadores da indústria do Sul do Brasil. Cadernos de Saúde Pública, Rio de Janeiro, v. 27, n. 2, p. 249-59, 2011. doi: https://doi.org/10.1590/S0102-311X2011000200006

THE EUROPEAN SOCIETY OF CARDIOLOGY [ESC]. European Guidelines on cardiovascular disease prevention in clinical practice (version 2012). European Journal of Preventive Cardiology, Londres, v. 19, n. 4, p. 585-891, 2012. doi: https:// doi.org/10.1177/2047487312450228

VIEIRA, V. R.; SILVA, J. V. P. Barreiras à prática de atividades físicas no lazer de brasileiros: revisão sistematizada. Revista Pensar a Prática, Goiânia, v. 22, n. 1, p. 1-22, 2019. doi: https://doi.org/10.5216/rpp.v22.54448

WHITTEMORE, R.; KNAFL, K. The integrative review: updated methodology. Journal of Advanced Nursing, Oxford, v. 52, n. 5, p. 546-53, 2005. doi: https://doi. org/10.1111/j.1365-2648.2005.03621.x

WORLD HEALTH ORGANIZATION [WHO]. Physical activity. Who, Geneva, 2020. Disponível em: https://www.who.int/en/news-room/fact-sheets/detail/physicalactivity/. Acesso em: 28 nov. de 2020. 
Gildiney Penaves de ALENCAR; Fabiana Maluf RABACOW; Alexandra Maria Almeida CARVALHO

WORLD HEALTH ORGANIZATION [WHO]. Global recommendations on physical activity for health. Geneva: WHO, 2010. 